Trinity University

Digital Commons@ Trinity

Mathematics Faculty Research

Mathematics Department

$5-2003$

\title{
Stability of Hyperbolic and Nonhyperbolic Fixed Points of One-Dimensional Maps
}

Fozi M. Dannan

Saber Elaydi

Trinity University, selaydi@trinity.edu

Vadim Ponomarenko

Trinity University

Follow this and additional works at: https://digitalcommons.trinity.edu/math_faculty

Part of the Mathematics Commons

\section{Repository Citation}

Dannan, F.M., Elaydi, S.N., \& Ponomarenko, V. (2003). Stability of hyperbolic and nonhyperbolic fixed points of one-dimensional maps. Journal of Difference Equations and Applications, 9(5), 449-457. doi:10.1080/1023619031000078315

This Post-Print is brought to you for free and open access by the Mathematics Department at Digital Commons @ Trinity. It has been accepted for inclusion in Mathematics Faculty Research by an authorized administrator of Digital Commons @ Trinity. For more information, please contact jcostanz@trinity.edu. 


\title{
Stability of Hyperbolic and Non Hyperbolic Fixed Points of One-Dimensional Maps
}

\author{
Fozi M. Dannan \\ Department of Mathematics, Faculty of Science, \\ Qatar University, Doha, Qatar \\ Saber N. Elaydi \\ Vadim Ponomarenko \\ Department of Mathematics, Trinity University, \\ San Antonio, Texas 78212
}

\section{Introduction}

At the sixth international conference on difference equations held in Augsburg, Germany, the second author was frequently asked about the stability of non-hyperbolic fixed points. It was rather surprising to learn that a complete stability theory for non-hyperbolic fixed points of one-dimensional maps is not known even to specialists in dynamical systems and difference equations. This is despite the fact that the study of the dynamics of onedimensional maps is central to many fields including discrete dynamical systems $[1,3,5,12]$, difference equations $[4,7,9,10]$ as well as differential equations via Poincaré [11] and Lorenz maps $[1,5,8]$.

Our main objective here is to present a complete theory for the stability of non-hyperbolic fixed points of one-dimensional continuous maps $f$. This would automatically provide a parallel theory for non hyperbolic periodic points by treating a $k$-periodic point $\tilde{x}\left(f^{k}(\tilde{x})=\tilde{x}\right)$ as a fixed point of the map $f^{k}$. Here $f^{k}$ denotes the $k$-time composition $f \circ f \circ \ldots \circ f$, while $f^{(k)}$ denotes the $k^{\text {th }}$ derivative of $f$, where $k \in Z^{+}$, the set of nonnegative integers.

Our proofs are simple and thus suitable for undergraduates with some calculus background. We hope that this paper will stir interest in teaching the 
intriguing dynamics of one-dimensional maps for all undergraduate science, mathematics and economics majors.

\section{Brief Review of the Literature}

Let $f: X \longrightarrow X$, where $X$ is an interval in $R$, be a continuous map and $x^{*} \in X$ be a fixed point of $f$, i.e., $f\left(x^{*}\right)=x^{*}$. Then we have the following definitions $[4,5,7]$ :

\section{Definition 2.1.}

i. $x^{*}$ is said to be stable if given $\varepsilon>0$, there exists $\delta>0$ such that $\left|x-x^{*}\right|<\delta$ implies $\left|f^{n}(x)-x^{*}\right|<\varepsilon$, for all $n \in Z^{+}$. $x^{*}$ is said to be unstable if it is not stable.

ii. $x^{*}$ is said to be attracting if there exists $\eta>0$ such that $\left|x-x^{*}\right|<\eta$ implies $\lim _{n \rightarrow \infty} f^{n}(x)=x^{*}$. Moreover, $x^{*}$ is globally attracting if $\eta=$ $\infty$.

iii. $x^{*}$ is said to be asymptotically stable if it is both stable and attracting. $x^{*}$ is said to be globally asymptotically stable if it is both stable and globally attracting.

iv. $x^{*}$ is said to be semi-asymptotically stable from the left (resp. right) if it is both

(a) stable from the left (resp. right), i.e., for every $\varepsilon>0$, there exists $\delta$ such that $x \in\left(x^{*}-\delta, x^{*}\right)\left(\right.$ resp. $\left.\left(x^{*}, x^{*}+\delta\right)\right)$ implies that $\left|f^{n}(x)-x^{*}\right|<\varepsilon$, and

(b) attracting from the left (resp. right), i.e., there exists $\eta>0$ such that $x \in\left(x^{*}-\eta, x^{*}\right)\left(\right.$ resp. $\left.\left(x^{*}, x^{*}+\eta\right)\right)$ implies that $\lim _{n \rightarrow \infty} f^{n}(x)=$ $x^{*}$.

The following theorem, found in $[4,5,14]$, summarizes what is known in the literature. Recall that a fixed point $x^{*}$ of a map is said to be hyperbolic if $\left|f^{\prime}\left(x^{*}\right)\right| \neq 1$, and non-hyperbolic if $\left|f^{\prime}\left(x^{*}\right)\right|=1$. The Schwarzian derivative of $f$ is defined as $S f(x)=\frac{f^{\prime \prime \prime}(x)}{f^{\prime}(x)}-\frac{3}{2}\left[\frac{f^{\prime \prime}(x)}{f^{\prime}(x)}\right]^{2}$. 
Theorem 2.2. The following statements hold true for a continuously differential hyperbolic map $f$.

i. If $\left|f^{\prime}\left(x^{*}\right)\right|<1$, then $x^{*}$ is asymptotically stable.

ii. If $\left|f^{\prime}\left(x^{*}\right)\right|>1$, then $x^{*}$ is unstable.

For non-hyperbolic maps, we have the following:

Theorem 2.3. The following statements hold true for $f \in C^{3}$.

i. If $f^{\prime}\left(x^{*}\right)=1$, then we have three cases to consider:

(a) If $f^{\prime \prime}\left(x^{*}\right) \neq 0$, then $x^{*}$ is semi-asymptotically stable from the left if $f^{\prime \prime}\left(x^{*}\right)>0$ and from the right if $f^{\prime \prime}\left(x^{*}\right)<0$.

(b) If $f^{\prime \prime}\left(x^{*}\right)=0$ and $f^{\prime \prime \prime}(x *)<0$, then $x^{*}$ is asymptotically stable.

(c) If $f^{\prime \prime}\left(x^{*}\right)=0$ and $f^{\prime \prime \prime}\left(x^{*}\right)>0$, then $x^{*}$ is unstable.

ii. If $f^{\prime}\left(x^{*}\right)=-1$, then we have two cases to consider:

(a) If $S f\left(x^{*}\right)<0$, then $x^{*}$ is asymptotically stable.

(b) If $S f\left(x^{*}\right)>0$, then $x^{*}$ is unstable.

Cull [2] and Rosenkranz [13] considered a special class of one-dimensional maps that they called population models, $x_{n+1}=f\left(x_{n}\right)$, where $f$ is a continuous map from $R^{+} \rightarrow R^{+}$with exactly one positive fixed point $x^{*}$ such that

i. $f(0)=0$

ii. $f(x) \begin{cases}>x & \text { for } 0<x<x^{*} \\ =x & \text { for } x=x^{*} \\ <x & \text { for } x>x^{*}\end{cases}$

For such models, Cull [2] considered the case when $f^{\prime}\left(x^{*}\right)=1$. Due to the complicated nature of the given stability conditions, we refrain from stating them here and refer the interested reader to Cull's paper. For global asymptotic stability of population models, Rosenkranz [13] gave the following interesting result. 
Theorem 2.4. Suppose that $f$ has no maximum in $\left(0, x^{*}\right)$, then $x^{*}$ is globally asymptotically stable. If $f$ has a maximum $x_{M} \epsilon\left(0, x^{*}\right)$, then $x^{*}$ is globally asymptotically stable if and only if $f^{2}(x)>x$ for all $x \in\left(x_{M}, x^{*}\right)$.

\section{Remark 2.5.}

i. Theorem 2.3 fails to address the situation when $f^{\prime}\left(x^{*}\right)=1$ and $f^{\prime \prime}\left(x^{*}\right)=$ $f^{\prime \prime \prime}\left(x^{*}\right)=0$ as in the case for $f(x)=x+(x-1)^{4}$ with $x^{*}=1$.

ii. Theorem 2.3 also fails to address the case when $f^{\prime}\left(x^{*}\right)=-1$ and $S f\left(x^{*}\right)=0$ as demonstrated by $f(x)=-x+2 x^{2}-4 x^{3}, x^{*}=0$.

iii. Theorem 2.3 does not address the extreme cases when $f^{\prime}\left(x^{*}\right)=1$ and $f^{(k)}\left(x^{*}\right)=0$ for $k \geq 2$, and $f^{\prime}\left(x^{*}\right)=-1$ and $g^{(k)}\left(x^{*}\right)=0$ for $k \geq 2$

\section{Global Stability of General Maps}

In this section, we divide the plane into eight regions $A_{i}, B_{i}, 1 \leq i \leq 4$ as depicted in the figure.

$$
\begin{aligned}
& A_{1}=\left\{(x, y): y \geq x, x \geq x^{*}\right\} \\
& A_{2}=\left\{(x, y): x^{*} \leq y \leq x\right\} \\
& A_{3}=\left\{(x, y):-x+2 x^{*} \leq y \leq x^{*}\right\} \\
& A_{4}=\left\{(x, y): y \leq-x+2 x^{*}, x \geq x^{*}\right\} \\
& B_{1}=\left\{(x, y):-x+2 x^{*} \leq y, x \leq x^{*}\right\} \\
& B_{2}=\left\{(x, y): x^{*} \leq y \leq-x+2 x^{*}\right\} \\
& B_{3}=\left\{(x, y): x \leq y \leq x^{*}\right\} \\
& B_{4}=\left\{(x, y): y \leq x \leq x^{*}\right\}
\end{aligned}
$$

Let $f: R \rightarrow R$ and $x^{*}$ be the only fixed point of $f$. Write $f$ as

$$
f(x)= \begin{cases}\psi(x) & \text { if } x \leq x^{*} \\ \varphi(x) & \text { if } x \geq x^{*}\end{cases}
$$

where $\psi\left(x^{*}\right)=\varphi\left(x^{*}\right)=x^{*}$ and both $\psi(x)$ and $\varphi(x)$ are continuous maps. The lines $y_{1}=x, y_{2}=2 x^{*}-x, y_{3}=x^{*}$, and $x=x^{*}$ divide the plane into the above-mentioned eight regions. 
Definition 3.1. If the set $\left\{(x, \psi(x)): x \leq x^{*}\right\} \subset B_{i}$, for some $i$, then we say that $\psi(x)$ stays in $B_{i}$. On the other hand, if the set $\{(x, \varphi(x)): x \geq$ $\left.x^{*}\right\} \subset A_{i}$, for some $i$, then we say that $\varphi(x)$ stays in $A_{i}$.

In the sequel, we will address the possible scenarios regarding the behavior of the map $f$ in the regions $A_{i}$ and $B_{i}, 1 \leq i \leq 4$.

Proposition 3.2. If $\varphi(x)$ stays in $A_{2}$ for all $x \in R$, then

i. $x^{*}$ is globally asymptotically stable if $\psi(x)$ stays in one of the regions $B_{1}, B_{2}$, or $B_{3}$.

ii. $x^{*}$ is semi-asymptotically stable from the right if $\psi(x)$ stays in $B_{4}$.

Proof. Let $x_{0}>x^{*}$. Then from the assumption $f(x)=\varphi(x)$ stays in the region $A_{2}$. Hence $x^{*} \leq f\left(x_{0}\right)<x_{0}$.

In the first case if $f\left(x_{0}\right)=x^{*}$, then $x^{*}$ is an eventually fixed point and the iterative process stops there. In the latter case, a decreasing sequence $\left\{f^{n}(x)\right\}$ is generated such that $\lim _{n \rightarrow \infty} f^{n}\left(x_{0}\right)=x^{*}$. This is true since if $\lim _{n \rightarrow \infty} f^{n}\left(x_{0}\right)=\tilde{x}$, then $\lim _{n \rightarrow \infty} f^{n+1}\left(x_{0}\right)=\tilde{x}=f(\tilde{x})$ and hence $\tilde{x}$ must be a fixed point of $f$. But then $\tilde{x}=x^{*}$, since $f$ has only one fixed point. On the other hand, if $x_{0}<x^{*}$ and $\psi(x)$ stays in $B_{1}$, then $\psi\left(x_{0}\right)=f\left(x_{0}\right)>x^{*}$. Consequently, $\lim _{n \rightarrow \infty} f^{n}\left(x_{0}\right)=x^{*}$.

The proofs of the remaining two cases and of (ii) are similar.

The remaining cases are now summarized in the following propositions.

Proposition 3.3. The following statements hold true.

i. If $\varphi(x)$ stays in $A_{3}$ for all $x \in R$, then $x^{*}$ is globally asymptotically stable if $\psi(x)$ stays in $B_{3}$; it is unstable if $\psi(x)$ stays in $B_{4}$.

ii. If $\varphi(x)$ stays in $A_{4}$ for all $x \in R$, then $x^{*}$ is unstable if $\psi(x)$ stays in $B_{3}$; it is globally asymptotically stable if $\psi(x)$ stays in $B_{4}$.

iii. If $\varphi(x)$ stays in $A_{1}$ for all $x \in X$, then $x^{*}$ is unstable if $\psi(x)$ stays in one of the regions $B_{1}, B_{2}$, or $B_{4}$; it is semi-asymptotically stable from the left if $\psi(x)$ stays in $B_{3}$. 


\section{Local Stability of Nonoscillatory Nonhy- perbolic Maps}

In this section, we will utilize propositions 3.3 and 3.2 to tackle the cases pertaining to $f^{\prime}\left(x^{*}\right)=1$ where $x^{*}$ is a fixed point of the map $f$.

Theorem 4.1. The following statements hold true:

i. Suppose that $f \in C^{2 k}$. If $f^{\prime}\left(x^{*}\right)=1$, and $f^{\prime \prime}\left(x^{*}\right)=\ldots=f^{(2 k-1)}\left(x^{*}\right)=$ 0 but $f^{(2 k)}\left(x^{*}\right) \neq 0$, then $x^{*}$ is semi-asymptotically stable from the left if $f^{(2 k)}\left(x^{*}\right)>0$ and from the right if $f^{(2 k)}\left(x^{*}\right)<0$.

ii. Suppose that $f \in C^{(2 k+1)}$. If $f^{\prime}\left(x^{*}\right)=1$, and $f^{\prime \prime}\left(x^{*}\right)=0 \ldots=$ $f^{(2 k)}\left(x^{*}\right)=0$ but $f^{(2 k+1)}\left(x^{*}\right) \neq 0$, then

(a) $x^{*}$ is (locally) asymptotically stable if $f^{(2 k+1)}\left(x^{*}\right)<0$,

(b) $x^{*}$ is unstable if $f^{(2 k+1)}\left(x^{*}\right)>0$

Proof of $(i)$. Let $q(x)=f(x)-x$ and let $\delta$ be a sufficiently small number. Then, by Taylor's Theorem

$$
q\left(x^{*}+\delta\right)=q\left(x^{*}\right)+q^{\prime}\left(x^{*}\right) \delta+\ldots+\frac{q^{(2 k-1)}\left(x^{*}\right)}{(2 k-1) !} \delta^{(2 k-1)}+\frac{q^{(2 k)}(\xi)}{(2 k) !} \delta^{2 k}
$$

Holds for some $x^{*}<\xi<x^{*}+\delta$, and hence

$$
q\left(x^{*}+\delta\right)=\frac{f^{2 k}(\xi)}{(2 k) !} \delta^{2 k}, x^{*}<\xi<x^{*}+\delta
$$

It is clear from Equation (1) that whenever $f^{(2 k)}\left(x^{*}\right)>0(<0)$, it follows that $q(x)>0(<0)$ in $\left(x^{*}-\delta, x^{*}+\delta\right)$ for a sufficiently small $\delta$. Applying Propositions 3.2 and 3.3 now yields the desired result.

Proof of (ii). Under the given assumption in part 2, and by using Taylor's theorem, we obtain

$$
q\left(x^{*}+\delta\right)=\frac{f^{(2 k+1)}(\xi)}{(2 n+1) !} \delta^{2 n+1}, x^{*}<\xi<x^{*}+\delta
$$

Now, from Equation (2), if $f^{(2 k+1)}\left(x^{*}\right)>0$, then $q(x)>0$ in $\left(x^{*}, x^{*}+\delta\right)$ and $q(x)<0$ in $\left(x^{*}-\delta, x^{*}\right)$, for a sufficiently small $\delta$. This implies, by 
Proposition 3.3, that $x^{*}$ is unstable. On the other hand, if $f^{(2 k+1)}\left(x^{*}\right)<0$, then $q(x)>0$ in $\left(x^{*}-\delta, x^{*}\right)$ and $q(x)<0$ in $\left(x^{*}, x^{*}+\delta\right)$, for a sufficiently small $\delta$. This implies by Proposition 3.2 that $x^{*}$ is asymptotically stable.

Recall the example $f(x)=x+(x-1)^{4}$. Here $x^{*}=1$ is a fixed point of $f$ with $f^{\prime}\left(x^{*}\right)=1, f^{\prime \prime}\left(x^{*}\right)=f^{\prime \prime \prime}\left(x^{*}\right)=0$ and $f^{(4)}\left(x^{*}\right)=24>0$. Hence by Theorem $4.1, x^{*}$ is semi-asymptotically stable from the left

\section{$5 \quad$ Local Stability of Oscillatory Nonhyperbolic Maps}

We now consider the case when $f^{\prime}\left(x^{*}\right)=-1$. A nice trick here is to look at the map $g(x)=f(f(x))=f^{2}(x)$.

\section{$5.1 \quad$ Results with $g(x)$}

Since $x^{*}$ is a fixed point of $f$, it must be a fixed point of $g$ and $g^{\prime}\left(x^{*}\right)=1$. Moreover, $g^{\prime \prime}\left(x^{*}\right)=0$ and $g^{\prime \prime \prime}\left(x^{*}\right)=2 S f\left(x^{*}\right)$ [4]. By results in [4, 14], we know that $x^{*}$ is asymptotically stable $\{$ unstable $\}$ under $g$ if, and only if, it is asymptotically stable $\{$ unstable $\}$ under $f$.

We can then apply the second half of Theorem 4.1 to get the following result.

Theorem 5.1. Suppose that $f \in C^{(2 k+1)}$ and $x^{*}$ is a fixed point of $f$ such that $f^{\prime}\left(x^{*}\right)=-1$. If $g^{\prime \prime}\left(x^{*}\right)=\ldots=g^{(2 k)}\left(x^{*}\right)=0$ and $g^{(2 k+1)}\left(x^{*}\right) \neq 0$, then

i. $x^{*}$ is asymptotically stable if $g^{(2 k+1)}\left(x^{*}\right)<0$ and

ii. $x^{*}$ is unstable if $g^{(2 k+1)}\left(x^{*}\right)>0$.

Observe that this strategy does not use the other part of Theorem 4.1 - where $x^{*}$ is semi-asymptotically stable under $g$. That is, the case where $f^{\prime}\left(x^{*}\right)=-1, g^{\prime \prime}\left(x^{*}\right)=\ldots=g^{(2 k-1)}\left(x^{*}\right)=0$ and $g^{(2 k)}\left(x^{*}\right) \neq 0$.

We now argue that this situation will never occur for analytic $f$.

Theorem 5.2. Let $f$ be analytic with $f^{\prime}\left(x^{*}\right)=-1$. Then, for some $k>1$,

i. If $g^{\prime \prime}\left(x^{*}\right)=\ldots=g^{(2 k-1)}\left(x^{*}\right)=0$, then $g^{(2 k)}\left(x^{*}\right)=0$. 
ii. $x^{*}$ cannot be semi-asymptotically stable under $g$.

Proof. By Taylor's Theorem, we have some small $\delta$ with

$$
\begin{aligned}
f\left(x^{*}+\delta\right) & =f\left(x^{*}\right)+\delta f^{\prime}\left(x^{*}\right)+\frac{\delta^{2} f^{\prime \prime}\left(x^{*}\right)}{2 !}+\ldots \\
& =x^{*}-\delta+0\left(\delta^{2}\right)
\end{aligned}
$$

Hence, for $x_{0}=x^{*}+\delta>x^{*}$, we have $f\left(x_{0}\right)<x^{*}$ and for $x_{0}=x^{*}-\delta<$ $x^{*}$, we have $f\left(x_{0}\right)>x^{*}$. In other words, for $x_{0} \in\left(x^{*}-\delta, x^{*}+\delta\right)$ either $f^{2 k}\left(x^{*}\right) \in\left(x^{*}, x^{*}+\delta\right)$ and $f^{2 k+1}\left(x^{*}\right) \in\left(x^{*}-\delta, x^{*}\right)$ for all $k \in Z^{+}$or $f^{2 k}\left(x^{*}\right) \in\left(x^{*}-\delta, x^{*}\right)$ and $f^{2 k+1}\left(x^{*}\right) \in\left(x^{*}, x^{*}+\delta\right)$ for all $k \in Z^{+}$.

Now, if $f^{2 k}\left(x_{0}\right) \rightarrow x^{*}$ as $k \rightarrow \infty$, then $f^{2 k+1}\left(x_{0}\right) \rightarrow x^{*}$ as $k \rightarrow \infty$. Hence either $x^{*}$ is asymptotically stable or $x^{*}$ is unstable and, more importantly, it cannot be semi-asymptotically stable.

These results using $g(x)$ are conclusive but not entirely satisfactory. For example, we return to $f(x)=-x+2 x^{2}-4 x^{3}$. To determine the stability of $f(x)$ at 0 , we need to find derivatives of $g(x)=-x+4 x^{2}-8 x^{3}+64 x^{5}-192 x^{6}+$ $384 x^{7}-384 x^{8}+256 x^{9}$. It turns out that $g^{5}(0)=7680$; hence, by Theorem 5.1, 0 is an unstable fixed point. However, this was computationally difficult, and we would like an analogue of Theorem 5.1 using only the derivatives of $f(x)$.

\subsection{Results to avoid $g(x)$}

We can avoid the need to calculate $g(x)$ by using another trick - Faà di Bruno's formula. A recent survey of the history of this marvelous result can be found in $[6]$.

Theorem 5.3 (Faà di Bruno). Let $f \in C^{n}$. Then $\frac{d^{n}}{d x^{n}} g(x)=\frac{d^{n}}{d x^{n}} f(f(x))=$ $\sum \frac{n !}{a_{1} ! a_{2} ! \cdots a_{n} !} f^{\left(a_{1}+a_{2}+\cdots+a_{n}\right)}(f(x))\left(\frac{f^{\prime}(x)}{1 !}\right)^{a_{1}}\left(\frac{f^{\prime \prime}(x)}{2 !}\right)^{a_{2}} \cdots\left(\frac{f^{(n)}(x)}{n !}\right)^{a_{n}}$

where the summation extends over all possible integer $a_{i}$ such that $0 \leq a_{i} \leq n$ and $n=a_{1}+2 a_{2}+3 a_{3}+\cdots+n a_{n}$.

We can combine Theorem 5.3 with Theorem 5.1 to make a stability test using only the derivatives of $f$. We know that $f^{\prime}\left(x^{*}\right)=-1$. We can use that in the formula, and we find the Schwarzian $g^{(3)}=-2 f^{(3)}-3\left(f^{(2)}\right)^{2}$. If this is nonzero, then Theorem 5.1 gives us an answer. If this is zero, then we know 
that $g^{(4)}=0$ by Theorem 5.2. We can now use $f^{\prime}=-1, f^{(3)}=-\frac{3}{2}\left(f^{(2)}\right)^{2}$ to find that $g^{(5)}=-2 f^{(5)}-15 f^{(2)} f^{(4)}+30\left(f^{(2)}\right)^{4}$.

This helps us right away with our example $f(x)=-x+2 x^{2}-4 x^{3}$. We have $f^{\prime}(x)=-1+4 x-12 x^{2}, f^{(2)}(x)=4-24 x, f^{(3)}(x)=-24$. Hence, $g^{(5)}=30(4)^{4}=7680$.

Theorem 5.4. If $f^{\prime}\left(x^{*}\right)=-1$ and $g^{\prime \prime}\left(x^{*}\right)=\cdots=g^{(2 k)}\left(x^{*}\right)=0$, then we can write $g^{(2 k+1)}\left(x^{*}\right)=-2 f^{(2 k+1)}\left(x^{*}\right)+p_{2 k+1}$, where $p_{2 k+1}$ is a polynomial in even derivatives of $f: f^{(2)}\left(x^{*}\right), f^{(4)}\left(x^{*}\right), \ldots f^{(2 k)}\left(x^{*}\right)$.

Proof. The summands in Theorem 5.3 only include terms in derivatives of $f$ up to the $(2 k+1)^{\text {th }}$. Furthermore, there are exactly two terms containing $f^{(2 k+1)}$ - one has $a_{1}=2 k+1,0=a_{2}=a_{3}=\cdots=a_{2 k+1}$, and the other has $0=a_{1}=a_{2}=\cdots=a_{n-1}, 1=a_{2 k+1}$. We can now prove the theorem by induction. The base case of $k=1$ is just the Schwarzian. For higher $k$ we can solve for the each of the odd derivatives of $f$ and successively substitute them into the formula of Theorem 5.3 until only even derivatives are left.

It is easy to calculate these $p_{2 k+1}$. We have:

$p_{3}=-3\left(f^{(2)}\right)^{2}$,

$p_{5}=-15 f^{(2)} f^{(4)}+30\left(f^{(2)}\right)^{4}$,

$p_{7}=-28 f^{(2)} f^{(6)}+945\left(f^{(2)}\right)^{3} f^{(4)}-\frac{4095}{2}\left(f^{(2)}\right)^{6}-35\left(f^{(4)}\right)^{2}$,

$p_{9}=-45 f^{(2)} f^{(8)}+4410\left(f^{(2)}\right)^{3} f^{(6)}-208845\left(f^{(2)}\right)^{5} f^{(4)}-210 f^{(4)} f^{(6)}+411075\left(f^{(2)}\right)^{8}+$ $15750\left(f^{(2)}\right)^{2}\left(f^{(4)}\right)^{2}$.

This gives us the following analog of Theorem 5.1.

Theorem 5.5. Let $f$ be an analytic function with $f^{\prime}\left(x^{*}\right)=-1$. Let $k$ be the smallest index where $f^{(2 k+1)}\left(x^{*}\right) \neq \frac{p_{2 k+1}\left(x^{*}\right)}{2}$. If $f^{(2 k+1)}\left(x^{*}\right)<\frac{p_{2 k+1}\left(x^{*}\right)}{2}$, then $f$ is unstable at $x^{*}$; otherwise $f^{(2 k+1)}\left(x^{*}\right)>\frac{p_{2 k+1}\left(x^{*}\right)}{2}$ and $f$ is asymptotically stable.

\subsection{Other results for oscillatory nonhyperbolic $f(x)$}

The previous theorems handle the cases of analytic (nonhyperbolic) $f$ where some $k^{\text {th }}$ derivative is nonzero, for $k>1$. If no such derivative is nonzero, then we have the following.

Remark 5.6. If $f^{\prime}\left(x^{*}\right)=1, f^{(k)}\left(x^{*}\right)=0$ for all $k>1$, and $f$ is analytic, then $f(x)=x$. Consequently, every point in the vicinity of $x^{*}$ is a fixed 
point and $x^{*}$ is thus stable but not asymptotically stable. If $f^{\prime}\left(x^{*}\right)=-1$, $g^{(k)}\left(x^{*}\right)=0$ for all $k>1$, and $f$ is analytic, then $g(x)=x$. Hence every point in the vicinity of $x^{*}$ is periodic of period 2 , and $x^{*}$ is again stable but not asymptotically stable.

If $\mathrm{f}$ is not analytic, we must revert to Proposition 3.3. The situation is much more involved as the following example demonstrates.

Example 5.7. Consider the maps $f_{1}(x)=x+e^{-x^{-2}}, f_{2}(x)=x+x e^{-x^{-2}}$, $f_{3}(x)=x-x e^{-x^{-2}}$, with $f_{i}(0)=0$. Each of these maps has $f_{i}^{\prime}(0)=-1$, and $f_{i}^{(k)}(0)=0$ for all $k>1$. However, the fixed point 0 is semi-asymptotically stable from the left, unstable, and asymptotically stable, respectively.

\section{Further Work}

In the course of this work several problems have suggested themselves. There appear to be deeper relationships between the derivatives of $f$ and $g$.

Conjecture 6.1. If $f^{\prime}\left(x^{*}\right)=1$ and $f^{\prime \prime}\left(x^{*}\right)=f^{\prime \prime \prime}\left(x^{*}\right)=\cdots=f^{(k-1)}\left(x^{*}\right)=0$, and $f^{(k)}\left(x^{*}\right) \neq 0$, then $g^{(k)}\left(x^{*}\right)=2 f^{(k)}\left(x^{*}\right)$.

Conjecture 6.2. If $f^{\prime}\left(x^{*}\right)=-1$ and $g^{\prime \prime}\left(x^{*}\right)=g^{\prime \prime \prime}\left(x^{*}\right)=\cdots=g^{(2 k-1)}\left(x^{*}\right)=$ 0 , then $g^{(2 k)}\left(x^{*}\right)=-\frac{1}{2} k(2 k-3) f^{\prime \prime}\left(x^{*}\right) g^{(2 k-1)}\left(x^{*}\right)$.

There is also some questions surrounding the $p_{2 k+1}$ from Theorem 5.4. What patterns are in those polynomials? It appears that the terms are of the following form, but the coefficients are a complete mystery.

Conjecture 6.3. In the notation of Theorem 5.4, the polynomials $p_{2 k+1}$ consists of terms $\alpha\left(f^{\left(a_{1}\right)}\right)^{b_{1}}\left(f^{\left(a_{2}\right)}\right)^{b_{2}} \cdots\left(f^{\left(a_{j}\right)}\right)^{b_{j}}$, where $2 \alpha \in \mathbb{Z}$ and $2 k=$ $\sum_{i} b_{i}\left(a_{i}-1\right)$.

Finally, we can construct polynomials $f$ that, locally, are square roots of $y=x$ to arbitrarily many derivatives. These $f$, incidentally, can be quite different from $y=-x$, the usual square root. We build $f$ from low-degree terms to high degree terms, by choosing the even derivatives arbitrarily, and setting the odd derivatives to satisfy Theorem 5.4. If we did this forever, we would get a power series instead of a polynomial. Can we choose the even derivatives to make the emerging power series converge? 


\section{References}

[1] Kathleen T. Alligood, Tim D. Sauer, and James A. Yorke. Chaos. Springer-Verlag, New York, 1997. An introduction to dynamical systems.

[2] Paul Cull. Stability of discrete one-dimensional population models. Bull. Math. Biol., 50(1):67-75, 1988.

[3] Robert L. Devaney. An introduction to chaotic dynamical systems. Addison-Wesley Publishing Company Advanced Book Program, Redwood City, CA, second edition, 1989.

[4] Saber N. Elaydi. An introduction to difference equations. SpringerVerlag, New York, second edition, 1999.

[5] Saber N. Elaydi. Discrete chaos. Chapman \& Hall/CRC, Boca Raton, FL, 2000.

[6] Warren P. Johnson. The curious history of Faà di Bruno's formula. Amer. Math. Monthy, 109(3):217-234, 2002.

[7] Walter G. Kelley and Allan C. Peterson. Difference equations. Harcourt/Academic Press, San Diego, CA, second edition, 2001. An introduction with applications.

[8] E. Lorenz. Deterministic nonperiodic flow. J Atmospheric Science, 20:130-141, 1963.

[9] R. M. May. Biological populations obeying difference equations: Stable points, stable cycles and chaos. Biol. Cybernet, 42:221-229, 1982.

[10] Ronald E. Mickens. Difference equations. Van Nostrand Reinhold Co., New York, second edition, 1990. Theory and applications.

[11] Richard K. Miller and Anthony N. Michel. Ordinary differential equations. Academic Press Inc. [Harcourt Brace Jovanovich Publishers], New York, 1982.

[12] Clark Robinson. Dynamical systems. CRC Press, Boca Raton, FL, second edition, 1999. Stability, symbolic dynamics, and chaos. 
[13] Gerd Rosenkranz. On global stability of discrete population models. Math. Biosci., 64(2):227-231, 1983.

[14] James T. Sandefur. Discrete dynamical systems. The Clarendon Press Oxford University Press, New York, 1990. Theory and applications. 